\title{
Value of Doppler evaluation of physically abnormal fistula: hemodynamic guidelines and access outcomes
}

\author{
Seong Cho, Yu-Ji Lee, and Sung-Rok Kim
}

Division of Nephrology, Department of Internal Medicine, Samsung Changwon Hospital, Sungkyunkwan University School of Medicine, Changwon, Korea
Received: September 19, 2016 Revised : October 27, 2016 Accepted: November 8, 2016

\section{Correspondence to}

Seong Cho, M.D.

Division of Nephrology, Department of Internal Medicine, Samsung Changwon Hospital, Sungkyunkwan University School of Medicine, 158 Paryong-ro, Masanhoewon-gu, Changwon 51353, Korea Tel: $+82-55-233-5140$

Fax: $+82-55-233-5149$

E-mail: chaecho67@gmail.com
Background/Aims: The strategy of access care at out center involves the use of ultrasound (USG) in case of physical examination (PE) abnormality. USG determines the need of angiography. This study investigated the possible association between the need for percutaneous transluminal angioplasty (PTA) and hemodynamic parameters of USG. The study also assessed the effects of this monitoring strategy on outcomes in comparison with a historical control.

Methods: A retrospective study of the medical records of 127 patients (65 PTA, 62 non-PTA) was conducted. Data were analyzed using logistic regression analysis and receiver operating characteristic curve. Fistula outcomes and intervention rates were calculated and compared with 100 historic controls.

Results: Logistic regression analysis showed that brachial artery flow volume (FV) $<612.9 \mathrm{~mL} / \mathrm{min}$ or brachial artery resistance index (RI) > 0.63 was independently associated with the need for PTA. This monitoring strategy showed an a reduction in thromboses $(0.02 \pm 0.11$ events/arteriovenous fistula [AVF]-year vs. $0.07 \pm$ 0.23 events/AVF-year, $p=0.046$ ), reduction in central venous catheter placement ( $0.01 \pm 0.05$ events/AVF-year vs. $0.06 \pm 0.22$ events/AVF-year, $p=0.010$ ), reduction in access loss ( $0.02 \pm 0.13$ events/AVF-year vs. $0.19 \pm 0.34$ events/AVF-year, $p=0.015$ ), and increase in access selective repair ( $0.49 \pm 0.66$ events/AVF-year vs. $0.21 \pm 0.69$ events/AVF-year, $p=0.003$ ), compared to historic control.

Conclusions: There was significant stenosis if brachial artery FV was $<612.9$ $\mathrm{mL} / \mathrm{min}$ or RI was $>0.63$ for PE abnormality. These parameters should be used as markers for assessing PTA risk in hemodialysis patients. Addition of USG to determine the need of angiography after detection of $\mathrm{PE}$ abnormality leads to decreases in access thrombosis, catheter placement, and access loss despite increasing access intervention rates compared to clinical monitoring.

Keywords: Ultrasonography, Doppler, color; Angioplasty; Vascular access

\section{INTRODUCTION}

Current guidelines recommend the development of specific monitoring programs for vascular access (VA) with the goal of early detection of vascular dysfunction, with the goal of reducing the percentage of thrombosis and number of central venous catheters [1,2]. But, currently available evidence indicates that surveillance-guided pre-emptive angioplasty may decrease the thrombosis rate, but does not increase the rate of overall VA survival 
[3]. Because of these conflicting results, a dialysis center relies on the experience and the competencies of the local nephrologists as well as the availability of other specialists at the site. Regular surveillance with ultrasound (USG) in addition to physical examination (PE) offers no additional benefits [4]. Nevertheless, USG has numerous effects on VA care including surveillance [5-7].

From 2011 at Samsung Changwon Hospital, the strategy was the routine USG confirmation of PE abnormality before angioplasty. Only dysfunctional fistula or abnormal PE finding fistula were evaluated by USG, compared to surveillance USG that evaluated all fistulas at regular intervals. USG hemodynamic parameters including brachial artery flow volume (FV) and resistance index (RI) are useful in objectively determining whether to perform percutaneous transluminal angioplasty (PTA).

The present study had two aims. One was to investigate a possible association between the need for PTA and hemodynamic parameters of USG. The other aim was to assess the effects of this monitoring strategy to investigate fistula thrombosis, abandonment, and intervention rates in comparison with a historical control.

\section{METHODS}

Before the study period (2007 to 2010), the historical control $(\mathrm{n}=100)$ was monitored with only clinical parameters. During the study period (2012 to 2015), augmentation test, arm elevation test, extent of thrill, and nature of bruit were tested weekly by the charge nurse. In case of PE abnormality or clinical abnormalities, such as negative pressure during dialysis, excessive post dialysis bleeding, and persistent cannulation difficulties, patients were evaluated using USG by the charge nephrologist. USG scanning of VA was performed with an 11-MHz linear probe (Vivid 7, GE Healthcare, Chicago, IL, USA). USG examinations were conducted before the hemodialysis (HD) session. Brachial artery FV, RI, and fistula stenosis diameter were measured (Fig. 1). In case of significant stenosis, patients were referred for access imaging. A total of 127 patients with renal failure treated with dialysis were included in this study. The PTA group (PTA) included patients who received an angiogram with $>50 \%$ stenosis detected (Fig. 2). The non-PTA group (N-PTA) included patients who did not need PTA

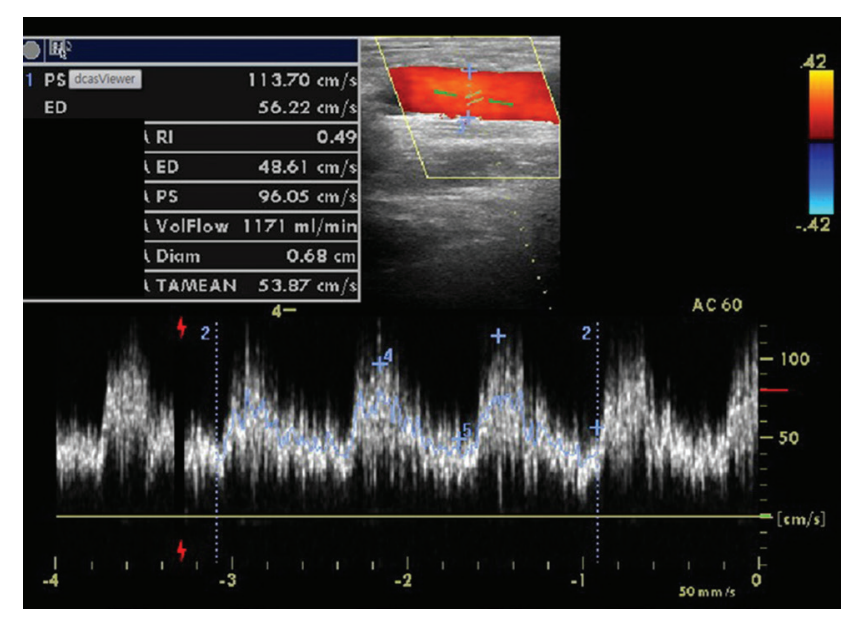

Figure 1. Doppler flow volume and resistance index measurements.

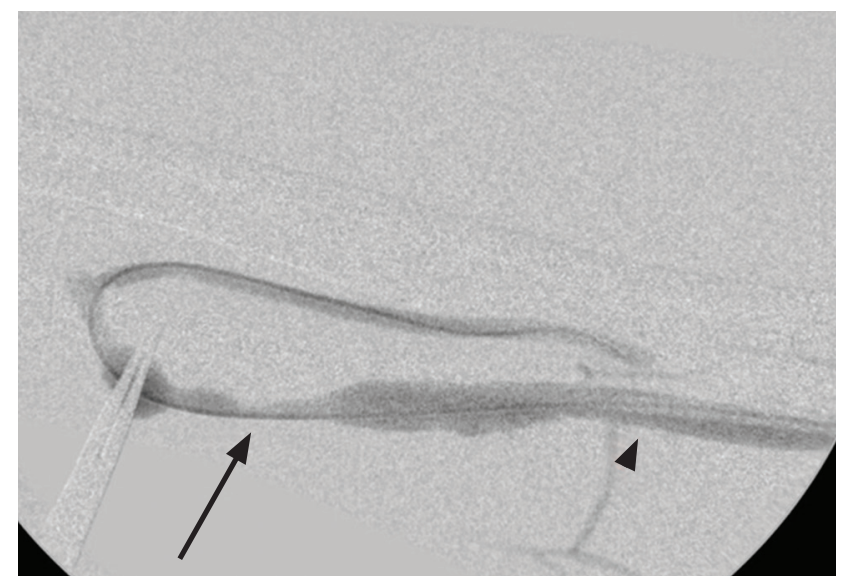

Figure 2. Angiographic diagnosis of stenosis (arrow); luminal diameter less than $50 \%$ compared to nonstenotic area (arrowhead).

following USG routine examination for internal control. N-PTA group had no abnormal PE findings and had a well-functioning fistula for more than 1 year. Maturating arteriovenous fistula (AVF; fistula's vintage was less than 3 months after creation) and maintenance AVF cases were all included. PE findings and angiographic film were reviewed. Data were collected on measured hemodynamic parameters; including brachial artery FV (mL/min) and brachial artery RI. Binomial logistic regression analysis was performed to explore the predictors of PTA. Logistic regression was performed to explore the relationships between the need for PTA and the remaining USG measures. If significant predictors were identified, the area under the receiver operating characteristic (ROC) curve (AUC) was calculated to assess the cutoff point for the 
risk of PTA. If the AUC was significant, the value, which yielded the combination of the highest true positive fraction (sensitivity) and the lowest false positive fraction (specificity), was used as the cutoff point for the measure.

The study also assessed the effects of this screening strategy to investigate access outcomes. Calculated parameters were rates of thrombosis, catheter requirement, access loss, and intervention. All were expressed as event/AVF-year. Thrombosis free patency was determined, and was defined as the interval between enrolment and access thrombosis. Primary access survival, defined as the interval from enrolment to first access intervention, cumulative survival, defined as the interval from enrollment to access loss by Kaplan-Meyer analysis. Follow-up period was calculated from study enrollment dates (incident patients: AVF operating dates; prevalent patients, January 1, 2007 in control, January 1, 2012 in study group) to follow-up end dates. Death, transplantation, transfer to other centers, or transfer to peritoneal dialysis cases were processed by censored data. For continuous variables, mean \pm standard deviation are reported. For categorical variables, percentages are reported. Baseline characteristics were compared using $t$ test and chi-square test as appropriate. All analyses were performed using the SPSS version 22.0 (IBM Co., Armonk, NY, USA) and the significance level was set at $p<0.05$ (two-tailed).
Informed consent was not required for this retrospective chart review as the Institutional Review Board approved the waiver of consent and exclusion of any access to identifiable private information.

\section{RESULTS}

The PTA group included 65 patients and the N-PTA group included 62 patients. Table 1 shows the baseline characteristics of the 127 enrolled patients. The average age in the PTA and N-PTA groups was similar (63.5 \pm 11.2 and $63.8 \pm 14.8$ years, respectively; $p>0.05)$. Females comprised $47.7 \%$ and $46.8 \%$ of the PTA and N-PTA group, respectively $(p>0.05)$. Average dialysis duration was shorter in the PTA (14.6 \pm 36.6 months) compared to N-PTA group $(36.7 \pm 40.8$ months, $p>0.000)$. Mean follow-up months from intervention or simple USG examination were similar between PTA group (27.0 \pm 15.9 months) and N-PTA group (27.0 \pm 17.1 months, $p>$ 0.05). Rates of maturating fistula was similar between two groups (PTA 9.2\%, N-PTA 6.5\%, p > 0.05). Diabetic nephropathy as a cause of etiology was in $55.4 \%$ in the PTA group compared to $29.0 \%$ in the N-PTA group $(p=$ $0.004)$. Brachial artery FV between the PTA and N-PTA group significantly differed (653.0 \pm 501.6 and 1,134.8 \pm $508.8 \mathrm{~mL} / \mathrm{min}, p=0.000$ ). Brachial artery RI was also significantly different $(0.62 \pm 0.14$ and $0.50 \pm 0.10, p=0.000)$.

Table 1. Background data of patients

\begin{tabular}{|c|c|c|c|}
\hline Variable & $\operatorname{PTA}(\mathrm{n}=65)^{\mathrm{a}}$ & Non-PTA $(n=62)^{b}$ & $p$ value \\
\hline Age, yr & $63.5 \pm 11.2$ & $63.8 \pm 14.8$ & NS \\
\hline Female sex & $31(47 \cdot 7)$ & $29(46.8)$ & NS \\
\hline Diabetes mellitus & $36(55 \cdot 4)$ & $18(29.0)$ & 0.004 \\
\hline AVF vintage, mon & $14.6 \pm 36.6$ & $36.7 \pm 40.8$ & 0.000 \\
\hline Follow-up, mon & $27.0 \pm 15.9$ & $27.0 \pm 17.1$ & NS \\
\hline Maturating $\mathrm{AVF}^{\mathrm{c}}$ & $6(9.2)$ & $4(6.5)$ & NS \\
\hline Upper arm fistula & $27(41.5)$ & $22(35 \cdot 5)$ & NS \\
\hline Brachial artery FV, mL/min & $653.0 \pm 501.6$ & $1134.8 \pm 508.8$ & 0.000 \\
\hline Brachial artery RI & $0.62 \pm 0.14$ & $0.50 \pm 0.10$ & 0.000 \\
\hline
\end{tabular}

Values are presented as mean \pm SD or number (\%).

PTA, percutaneous transluminal angioplasty; NS, not significant; AVF, arteriovenous fistula; FV, flow volume; RI, resistive index.

${ }^{a}$ Patients group who had angioplasty.

${ }^{\mathrm{b}}$ Patients group who had not angioplasty.

${ }^{\mathrm{c}}$ Fistula's vintage was less than 3 months after creation. 
Table 2. Logistic regression analysis of risk factors correlated with percutaneous transluminal angioplasty

\begin{tabular}{lccc}
\hline Variable & p value & Odds ratio & $95 \%$ CI \\
\hline Brachial artery FV & 0.040 & 0.999 & $0.998-1.000$ \\
Brachial artery RI & 0.003 & 1.066 & $1.021-1.112$ \\
\hline
\end{tabular}

Multiple adjusted $R^{2}=0.396(p<0.001)$.

CI, confidence Interval; FV, flow volume; RI, resistive index.

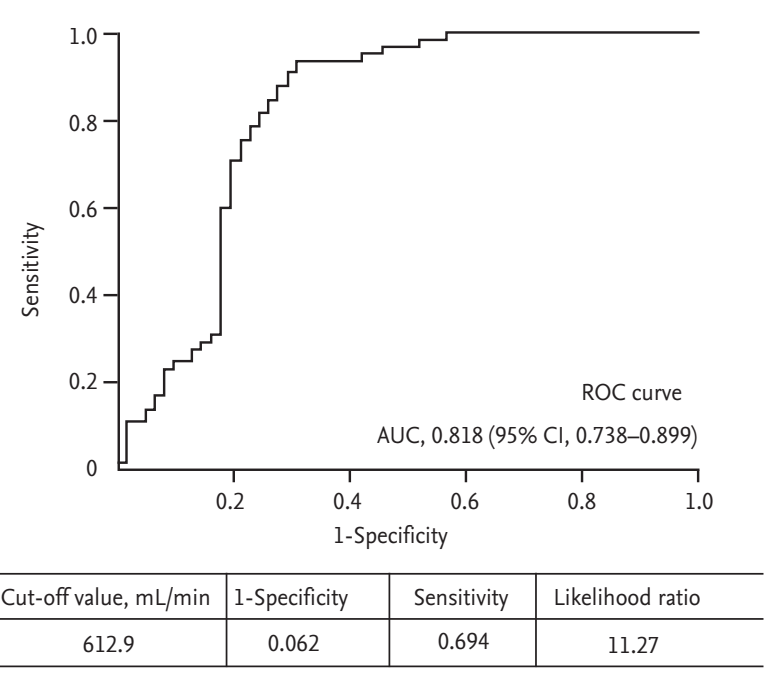

Figure 3. Receiver operating characteristic (ROC) for brachial artery flow volume to discriminate percutaneous transluminal angioplasty. AUC, area under the ROC curve; CI, confidence interval.

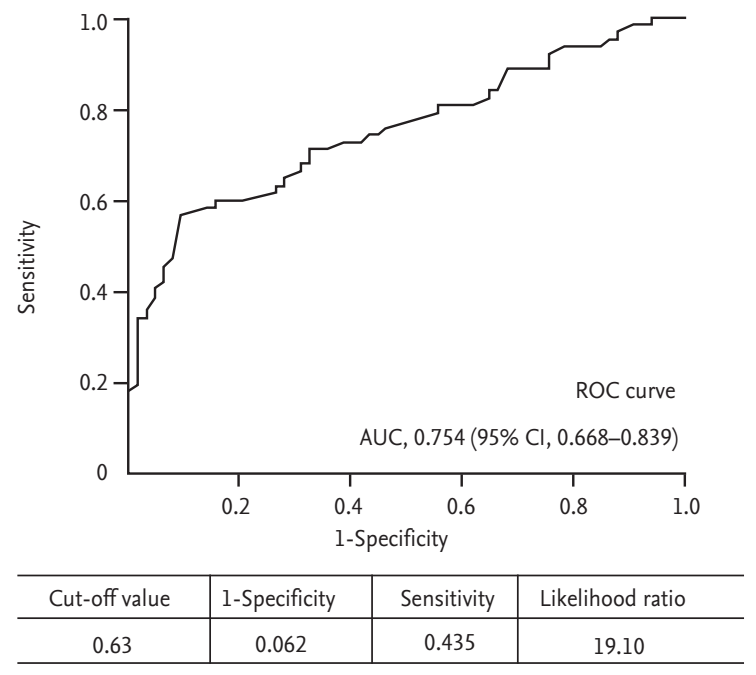

Figure 4. Receiver operating characteristic (ROC) for brachial artery resistance index to discriminate percutaneous transluminal angioplasty. AUC, area under the ROC curve; CI, confidence interval.

Table 3. Patient and AVF characteristics between historic control (C) and study group (S)

\begin{tabular}{|c|c|c|c|}
\hline Characteristic & $C(n=100)$ & $\mathrm{S}(\mathrm{n}=127)$ & $p$ value \\
\hline Study period, yr & $2007-2010$ & $2012-2015$ & \\
\hline Age, yr & $60.9 \pm 13.7$ & $63.6 \pm 13.1$ & NS \\
\hline Female sex & $46(48.7)$ & $60(46.3)$ & NS \\
\hline Diabetes mellitus & $45(42.6)$ & $54(46.3)$ & NS \\
\hline AVF vintages, mon & $54.1 \pm 38.6$ & $25.3 \pm 32.5$ & 0.000 \\
\hline Maturating $\mathrm{AVF}^{\mathrm{a}}$ & $7(7.0)$ & $10(7 \cdot 9)$ & NS \\
\hline Follow-up, mon & $36.7 \pm 14.5$ & $27.0 \pm 16.4$ & 0.000 \\
\hline Completed study & $46(46.0)$ & $102(80.3)$ & \\
\hline Deaths & $30(30.0)$ & $11(8.7)$ & \\
\hline Transplantation & $4(4 \cdot 0)$ & $6(4 \cdot 7)$ & \\
\hline Transfer to other centers & $18(18.0)$ & $8(6.3)$ & \\
\hline Transfer to PD & $2(2.0)$ & o & \\
\hline
\end{tabular}

Values are presented as mean $\pm \mathrm{SD}$ or number (\%).

AVF, arteriovenous fistula; NS, not significant; PD, peritoneal dialysis.

${ }^{a}$ Fistula's vintage was less than 3 months after creation. 
Table 4. Individual access outcomes between historic control (C) and study group (S)

\begin{tabular}{|c|c|c|c|}
\hline Variable & $\mathrm{C}(\mathrm{n}=100)$ & $\mathrm{S}(\mathrm{n}=127)$ & $p$ value \\
\hline Total stenosis repair & 40 & 122 & \\
\hline Elective stenosis repair & $12(30.0)$ & $111(91.0)$ & $0.000^{\mathrm{a}}$ \\
\hline Emergency stenosis repair & $28(70.0)$ & $11(9.0)$ & \\
\hline Intervention (patients no.) & 25 & 65 & $0.353^{\mathrm{a}}$ \\
\hline One intervention & $16(64.0)$ & $34(52.3)$ & \\
\hline Two or more interventions & $9(36.0)$ & $31(47 \cdot 7)$ & \\
\hline Total stenosis repair rate ${ }^{b}$ & $0.21 \pm 0.69$ & $0.49 \pm 0.66$ & 0.003 \\
\hline Thrombosis rates ${ }^{\mathrm{b}}$ & $0.07 \pm 0.23$ & $0.02 \pm 0.11$ & 0.046 \\
\hline Catheter insertion rates ${ }^{b}$ & $0.06 \pm 0.22$ & $0.01 \pm 0.05$ & 0.010 \\
\hline Access loss rates $^{b}$ & $0.10 \pm 0.34$ & $0.02 \pm 0.13$ & 0.015 \\
\hline
\end{tabular}

Values are presented as number (\%) or mean \pm SD.

${ }^{\mathrm{a} C h i}$-square test.

${ }^{b}$ Events/patients arteriovenous fistula years.

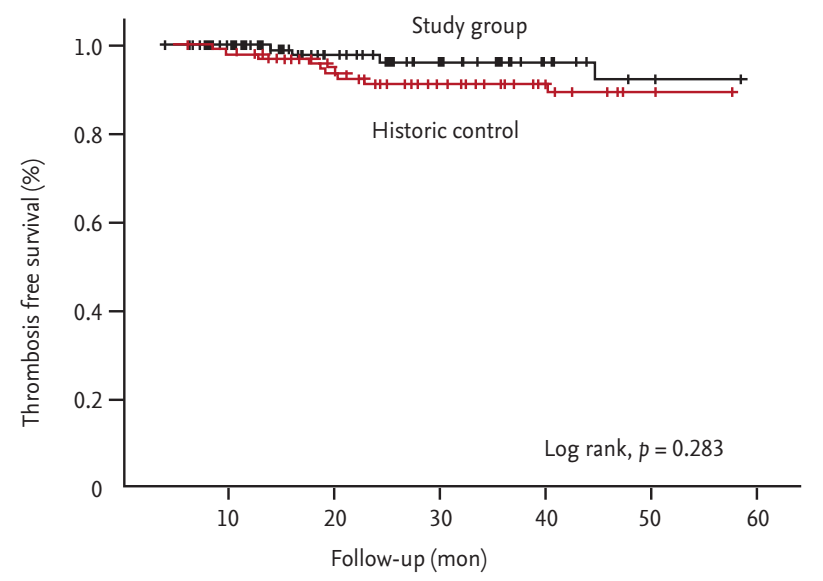

Figure 5. Thrombosis free survival. The graphs show the unadjusted thrombosis free survival as of enrollment. The Kaplan-Meier analysis showed that thrombosis free survival was better in study group (black colored dashed line) than in historic control (red colored dashed line) but statistically nonsignificant.

Table 2 provides the results of the logistic regression analysis of the odds ratio correlated with PTA. Brachial artery FV $(p<0.040)$, and brachial artery RI $(p<0.003)$ were independently associated with PTA, with a multiple adjusted $R^{2}$ of $0.396(p<0.001)$. In the ROC curve for brachial artery FV to predict PTA, the AUC was 0.818 (95\% confidence interval [CI], 0.738 to 0.899 ) (Fig. 3). The optimal cutoff point for brachial artery FV was $612.9 \mathrm{~mL} /$ min (1-specificity 0.062, sensitivity 0.694, and likelihood ratio 11.27). In ROC curve for brachial artery RI to predict

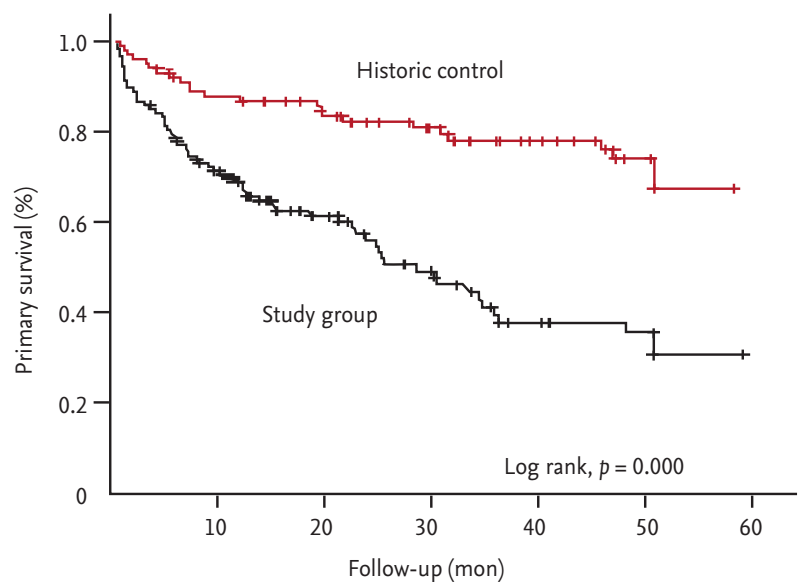

Figure 6. Primary survival. The graphs show the primary survival as of enrollment. The Kaplan-Meier analysis showed that primary access survival was significantly lower in study group (black colored dashed line) than in historic control (red colored dashed line) with the log rank test.

PTA, the AUC was 0.754 (95\% CI, 0.668 to 0.839 ) (Fig. 4). The optimal cutoff point for brachial artery RI was 0.63 (1-specificity 0.062, sensitivity 0.435 , and likelihood ratio 19.10). The results of access outcomes between study group (S) and historic control(C) were compared. Patient and AVF characteristics are provided in Table 3. The two groups were well matched for baseline parameters of age ([C] $60.9 \pm 13.7$ years vs. [S] $63.6 \pm 13.1$ years, $p>0.05$ ) and female sex ([C] $46.0 \%$ vs. [S] 47.2\%, $p>0.05$ ), but AVF vintage at enrollment was shorter in $\mathrm{S}$ compared to $\mathrm{C}$ 


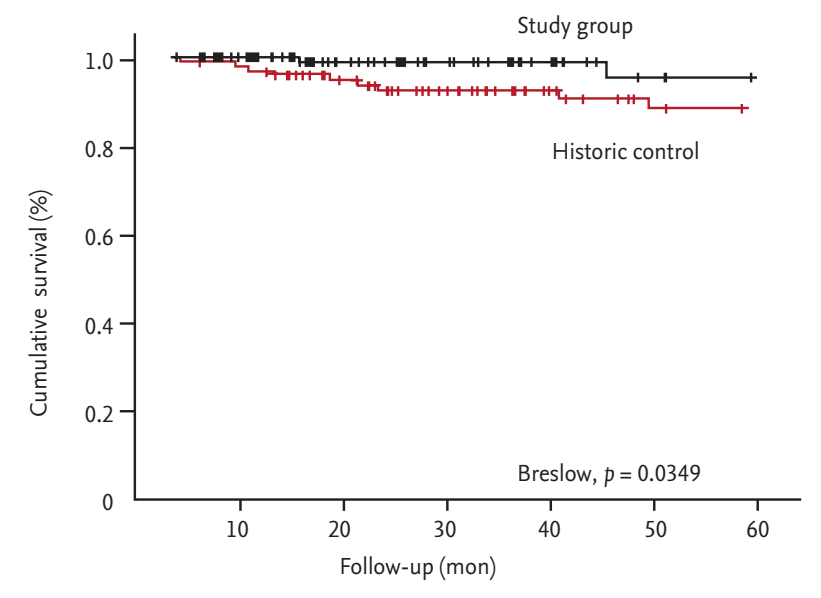

Figure 7. Cumulative survival. The graphs show the cumulative survival as of enrollment. The Kaplan-Meier analysis showed that access survival was significantly better in study group (black colored dashed line) than in historic control (red colored dashed line) with the Breslow test.

$(25.3 \pm 32.5$ months vs. $54.1 \pm 38.6$ months, $p=0.000)$. The length of follow-up was shorter in S compared to C (27.0 \pm 16.4 months vs. $38.6 \pm 14.5$ months, $p=0.000)$. In $S, 25$ cases were censored data (11 death, six transplantation, and eight transfer to other centers). In C, 54 cases were censored data (30 death, four transplantation, 18 transfer to other centers, and two transfer to peritoneal dialysis). Only autogeneous fistula was included in both groups. A total 122 angioplasties $(0.49 \pm 0.66$ events/AVF-year) were performed in the $\mathrm{S}$ group compared to 40 angioplasties ( $0.21 \pm 0.69$ events/AVF-year) in the C group $(p=0.003)$. There was elective repair of 111 cases of stenosis in the S group (111/123, 91.0\%) and 11 stenosis were emergently repaired. In C, 12 stenosis (12/40, 30.0\%) were electively repaired and 28 stenosis were emergently repaired $(p$ $=0.000$ ). Proportions of patient with consecutive multiple angioplasties was higher in $\mathrm{S}$ compared to $\mathrm{C}$ but statistically not significant $(16$ [64.0\%] vs. 34 [52.3\%], $p=$ 0.353) (Table 4). Nine AVFs (0.07 \pm 0.23 events/AVF-year) thrombosed in $\mathrm{C}$ and four AVFs (0.02 \pm 0.11 events/AVFyear) in $S(p=0.046)$. Twelve temporary central venous catheters were placed in $\mathrm{C}(0.06 \pm 0.22$ events/AVF-year) and two ( $0.01 \pm 0.05$ events/AVF-year) in $\mathrm{S}(p=0.010)$. Nine AVFs ( $0.10 \pm 0.34$ events/AVF-year) failed in $\mathrm{C}$ and two AVFs (0.02 \pm 0.13 events/AVF-year) in $S(p=0.015)$. The Kaplan-Meier analysis showed that thrombosis free survival was better in $\mathrm{S}$ than in $\mathrm{C}$ but statistically nonsignificant (log rank, $p=0.2830$ ) (Fig. 5), showed that primary access survival was significantly lower in $\mathrm{S}$ than in $\mathrm{C}(\log$ rank, $p=0.000$ ) (Fig. 6) and showed that cumulative access survival was significantly better in $\mathrm{S}$ than in $\mathrm{C}$ (Breslow test, $p=0.0362$ ) (Fig. 7).

\section{DISCUSSION}

Maintenance of well-functioning VA is essential for the smooth continuation of HD treatment. We investigated the correlations between the VA screening method using USG and the presence or absence of PTA. In a study from Japan, the target was insufficient blood flow during dialysis (e.g., $<200 \mathrm{~mL} / \mathrm{min}$ ). This target was detected by brachial artery FV $<354 \mathrm{~mL} / \mathrm{min}$, or RI $>0.61$ [8]. The authors recommended angioplasty at the lower target compared to the Kidney Disease Outcomes Quality Initiative guideline of 500 to $600 \mathrm{~mL} / \mathrm{min}$ [1] to prevent multiple, repeated angioplasty procedures that are expensive and can promote neointimal hyperplasia and restenosis during balloon angioplasty. After implementation of this FV guideline, angioplasty frequency decreased without affecting access longitivity. This hemodynamic parameter corresponds to the European Best Practice Guideline [2] that recommends preemptive angioplasty at $300 \mathrm{~mL} / \mathrm{min}$ in the fistula. Multiple repeated angioplasties were more common compared to clinical monitoring group in this study but statistically not significant. Another Japanese study involved satellite clinics, where prompt angioplasty was seldom possible. Angioplasty was done when the FV level was 665 $\mathrm{mL} / \mathrm{min}$ [9], which was higher than the aforementioned study [8]. Hemodynamic guidelines can differ according to the objectives of access care, such as reduction of sulfurous angioplasties or to decrease minor stenosis. In this study, the target of angioplasties were included because of difficulty with the actual dialysis procedure and to address PE abnormality without interrupting dialysis treatment. Therefore, more mild stenotic cases were included compared to studies addressing only flow insufficiency. At this FV level $(612.9 \mathrm{~mL} / \mathrm{min})$ or RI (0.63), significant stenosis (> 50\%) was detected in the angiogram in response to PE abnormality. These FV or RI levels were required to detect asymptomatic stenosis that requires treatment when the dialysis sessions 
go smoothly in our center. If angiographic criteria are changed to more than $75 \%$ luminal reduction compared to $50 \%$ luminal reduction, contemporary hemodynamic guidelines may be changed. In one review article [10], timing of USG examination of VA was related to brachial artery FV, with an interval of 12 months for FV 800 to 1,200 mL/min, 6 months for $\mathrm{FV}$ of 600 to $800 \mathrm{~mL} / \mathrm{min}$, and 1 month for FV $<600 \mathrm{~mL} / \mathrm{min}$. In addition to $\mathrm{PE}$ abnormality and clinical abnormalities, flow monitoring [11,12], pressure monitoring [13], and Doppler surveillance [14-20] have been proposed and implemented for evaluation of VA. Without functional severity criteria, USG surveillance leads to many unnecessary angioplasty procedures based on simple morphologic data, which in turn leads to more aggressive neointimal hyperplasia that results in failure of surveillance [14-20]. Addition of functional severity to morphologic stenosis was reported to achieve better long term survival [21] compared to solely a morphologic-guided strategy [14-20]. Therefore, using brachial artery FV or RI as an objective guide for intervention is essential. Clinical monitoring remains the backbone of any VA program. Lomonte et al. [22] strongly advocate the adoption of a VA surveillance program based on Doppler. Flow monitoring does not detect the exact site of stenosis. Doppler is very important in a patient-centered VA evaluation, because it allows the appropriate management of all aspects of VA care including vascular mapping, maturation evaluation, and surveillance. USG shortens angioplasty times because it provides information about the stenosis site [23]. Therefore, more clinical needs are met by use of USG. Access outcome monitoring incorporating thrombotic rate and cumulative survival is necessary to examine the monitoring efficacy of a clinical center. Prior USG surveillance results have been confusing [14-20]. The present findings indicate that introduction of USG at VA care can decrease thrombotic loss and increase cumulative access survival compared to only clinical monitoring, despite the increased rate of elective angioplasty (i.e., decreased primary patency compared to historic control). Our study population thrombosis rate $(0.02 \pm 0.11$ event/AVF-year) compares favorably with the lowest thrombosis rates reported in the literature using quality assurance surveillance, which ranges from 0.04 to 0.10 thromboses/AVF-year [12,24-29]. The thrombosis rate of $0.07 \pm 023$ event/AVF-year in our control arm was also at the lower end of the range reported in observational studies with bedside clinical monitoring ( 0.03 to 0.17 thromboses/AVF-year) [12,24-29]. The cumulative access survival was prolonged compared to the historic control, but was also comparable to Italian [18] and Korean [30] access trials that performed regular FV surveillance (Qa). High proportions (100\%) of autogenous fistula, more matured fistula compared to maturing fistula, rigorous $\mathrm{PE}$ and speedy action to repair were factors associated with low thrombosis and high survival rates in this study. The present access intervention rate of $0.49 \pm 0.66 /$ AVF-year was significantly higher compared to the control group $(0.21 \pm 0.69)$, but was at the higher end of the range reported in the literature using Qa surveillance [29,30]. This reflects the many interventions performed according to this strategy compared to clinical examination or Qa monitoring. Direct morphology evaluation was possible in USG evaluation compared to Qa studies, and could have been a possible reason for more pectoral decision-making. If more reduced hemodynamic guidelines, such as the European Best Practice Guideline [2] are introduced (FV $<300 \mathrm{~mL} / \mathrm{min}$ ), the intervention rate may decrease. If USG surveillance is routinely adopted in all fistula patients, the result could be more and effort, despite the lack of proven beneficial effects. But, in selected patients, such as these with abnormal PE findings, USG could be a more cost effective treatment. The caveat with this is that the approach is possible only when regular PE and clinical evaluation are scrupulous and the center experiences a high percentage of autogenous fistulas, because grafts can more commonly thrombose unexpectedly.

The present results confirm the prior report that systemic USG evaluation can decrease emergency intervention and reduced X-ray exposure times [23]. Most cases of intervention in the study group were elective whereas most historic interventions were emergent. We recognize that our study has important limitations. This study was retrospective nature, was compared data to the historical control and was single center study. Therefore this study had a selection bias. Our study was possibly underpowered for the detection of differences in access survival, due to the unexpectedly low failure rate in the control group. We are aware that our study cannot provide a definitive answer on the role of this strategy in AVF and that further better designed trials 
need to be undertaken.

In conclusion, there was significant stenosis if brachial artery $\mathrm{FV}<612.9 \mathrm{~mL} / \mathrm{min}$ or $\mathrm{RI}>0.63$ in the presence of PE or clinical abnormality. These parameters could be used as markers for assessing PTA risk in HD patients. The strategy of addition of USG FV or RI to determine the need for angiography after detection of PE abnormality leads to decreases in access thrombosis, catheter placement, and access loss despite increasing access intervention rates compared to only clinical monitoring. More efforts to decrease the frequency of angioplasty frequencies are needed, such as angiographic criteria change or results when reduced hemodynamic guidelines are implemented.

\section{KEY MESSAGE}

1. There was significant stenosis if brachial artery flow volume $(\mathrm{FV})<612.9 \mathrm{~mL} / \mathrm{min}$ or resistance index $(\mathrm{RI})>0.63$ in the presence of physical examination (PE) or clinical abnormality. These parameters could be used as markers for assessing percutaneous transluminal angioplasty risk in hemodialysis patients.

2. The strategy of addition of ultrasound FV or RI to determine the need for angiography after detection of PE abnormality leads to decreases in access thrombosis, catheter placement, and access loss despite increasing access intervention rates compared to only clinical monitoring.

\section{Conflict of interest}

No potential conflict of interest relevant to this article was reported.

\section{REFERENCES}

1. National Kidney Foundation K/DOQI. Clinical practice guidelines for vascular access: 2006 updates [internet]. New York (NY): National Kidney Foundation, c2006 [cited 2017 Mar 24]. Available from: http://www2.kidney.org/professionals/KDOQI/guideline_upHD_PD_VA/.

2. Tordoir J, Canaud B, Haage P, et al. European best practice guidelines on vascular access. Nephrol Dial Trans- plant 2007;22(Suppl 2):ii88-iin17.

3. Ravani P, Quinn RR, Oliver MJ, et al. Preemptive correction of arteriovenous access stenosis: a systematic review and meta-analysis of randomized controlled trials. Am J Kidney Dis 2016;67:446-460.

4. Malik J, Kudlicka J, Novakova L, Adamec J, Malikova H, Kavan J. Surveillance of arteriovenous accesses with the use of duplex Doppler ultrasonography. J Vasc Access 2014;15 Suppl 7:S28-S32.

5. Guedes Marques M, Ibeas J, Botelho C, Maia P, Ponce P. Doppler ultrasound: a powerful tool for vascular access surveillance. Semin Dial 2015;28:206-210.

6. Wong CS, McNicholas N, Healy D, et al. A systematic review of preoperative duplex ultrasonography and arteriovenous fistula formation. J Vasc Surg 2013;57:1129-1133.

7. Ko SH, Bandyk DF, Hodgkiss-Harlow KD, Barleben A, Lane J 3rd. Estimation of brachial artery volume flow by duplex ultrasound imaging predicts dialysis access maturation. J Vasc Surg 2015;61:1521-1527.

8. Sato T, Tsuboi M, Onogi T, et al. Standard procedures of endovascular treatment for vascular access stenosis in our facility - clinical usefulness of ultrasonography. J Vasc Access 2015;16 Suppl 10:S34-S37.

9. Sato T, Tsuboi M, Onogi T, Miwa N, et al. Standard procedures of endovascular treatment for vascular access stenosis in our facility: clinical usefulness of ultrasonography. J Vasc Access 2015;16 Suppl 10:S34-S37.

10. Mudoni A, Caccetta F, Caroppo M, et al. Echo color Doppler ultrasound: a valuable diagnostic tool in the assessment of arteriovenous fistula in hemodialysis patients. J Vasc Access 2016;17:446-452.

11. Tessitore N, Lipari G, Poli A, et al. Can blood flow surveillance and pre-emptive repair of subclinical stenosis prolong the useful life of arteriovenous fistulae? A randomized controlled study. Nephrol Dial Transplant 2004;19:2325-2333.

12. Polkinghorne KR, Lau KK, Saunder A, Atkins RC, Kerr PG. Does monthly native arteriovenous fistula blood-flow surveillance detect significant stenosis: a randomized controlled trial. Nephrol Dial Transplant 2006;21:24982506.

13. Sands JJ, Jabyac PA, Miranda CL, Kapsick BJ. Intervention based on monthly monitoring decreases hemodialysis access thrombosis. ASAIO J 1999;45:147-150.

14. Malik J, Slavikova M, Svobodova J, Tuka V. Regular ultrasonographic screening significantly prolongs patency of 
PTFE grafts. Kidney Int 2005;67:1554-1558.

15. Ram SJ, Work J, Caldito GC, Eason JM, Pervez A, Paulson WD. A randomized controlled trial of blood flow and stenosis surveillance of hemodialysis grafts. Kidney Int 2003;64:272-80.

16. Robbin ML, Oser RF, Lee JY, Heudebert GR, Mennemeyer ST, Allon M. Randomized comparison of ultrasound surveillance and clinical monitoring on arteriovenous graft outcomes. Kidney Int 2006;69:730-735.

17. Dossabhoy NR, Ram SJ, Nassar R, Work J, Eason JM, Paulson WD. Stenosis surveillance of hemodialysis grafts by duplex ultrasound reduces hospitalizations and cost of care. Semin Dial 2005;18:550-557.

18. Mayer DA, Zingale RG, Tsapogas MJ. Duplex scanning of expanded polytetrafluoroethylene dialysis shunts: impact on patient management and graft survival. Vasc Endovasc Surg 1993;27:647-658.

19. Lumsden AB, MacDonald MJ, Kikeri D, Cotsonis GA, Harker LA, Martin LG. Prophylactic balloon angioplasty fails to prolong the patency of expanded polytetrafluoroethylene arteriovenous grafts: results of a prospective randomized study. J Vasc Surg 1997;26:382-390

20. Bay WH, Henry ML, Lazarus JM, Lew NL, Ling J, Lowrie EG. Predicting hemodialysis access failure with color flow Doppler ultrasound. Am J Nephrol 1998;18:296-304.

21. Malik J, Tuka V, Chytilova E, Mokrejsova M, Masek M, Slavikova M. Low-flow polytetrafluoroethylene accesses: ultrasound surveillance and preemptive interventions ensure long-term patency. Kidney Blood Press Res 2010;33:181-185.

22. Lomonte C, Meola M, Petrucci I, Casucci F, Basile C. The key role of color Doppler ultrasound in the work-up of hemodialysis vascular access. Semin Dial 2015;28:211-215.

23. Matsui S, Nakai K, Taniguchi T, et al. Systematic evaluation of vascular access by color-Doppler ultrasound decreased the incidence of emergent vascular access intervention therapy and X-ray exposure time: a single-center observational study. Ther Apher Dial 2012;16:169-172.

24. Schwab SJ, Oliver MJ, Suhocki P, McCann R. Hemodialysis arteriovenous access: detection of stenosis and response to treatment by vascular access blood flow. Kidney Int 2001;59:358-362.

25. Wijnen E, Planken N, Keuter X, et al. Impact of a quality improvement programme based on vascular access flow monitoring on costs, access occlusion and access failure. Nephrol Dial Transplant 2006;21:3514-3519.

26. McCarley P, Wingard RL, Shyr Y, Pettus W, Hakim RM, Ikizler TA. Vascular access blood flow monitoring reduces access morbidity and costs. Kidney Int 2001;60:1164-1172.

27. Lok CE, Bhola C, Croxford R, Richardson RM. Reducing vascular access morbidity: a comparative trial of two vascular access monitoring strategies. Nephrol Dial Transplant 2003;18:1174-1180.

28. Shahin H, Reddy G, Sharafuddin M, Katz D, Franzwa BS, Dixon BS. Monthly access flow monitoring with increased prophylactic angioplasty did not improve fistula patency. Kidney Int 2005;68:2352-2361.

29. Roca-Tey R, Samon Guasch R, Ibrik O, et al. Vascular access surveillance with blood flow monitoring: a prospective study with 65 patients. Nefrologia 2004;24:246-252.

30. Choi SJ, Park MY, Kim JK, Hwang SD, Her K, Won Y. Impact of initial blood flow on outcomes of vascular access in hemodialysis patients. Kidney Res Clin Pract 2012;31:151-156. 